\title{
SECURING HUMAN RIGHTS THROUGH PROMOTION AND TRAINING
}

\author{
Sarah Cooke, Former Director of the British Institute of Human \\ Rights
}

\section{The Need for Innovative Approaches}

The Human Rights Act was heralded by the government as a tool that would not only give people redress in the domestic courts for the breaches of the European Convention on Human Rights, but would help change the relationship between the state and individual more widely, creating a "culture of rights". It became quickly apparent, however, that, without some concerted action, a culture based on respect for human rights was not going to emerge on its own by virtue of having a Human Rights Act on the statute book.

An early indicator of this was the predictable (prior to the Human Rights Act coming into force) and observable (once it had done so) fact that socially and economically excluded people were the least likely section of the population to achieve real benefit from the Act. This is despite the self-evident fact that it is precisely these people who are most likely to suffer an infringement of their human rights. ${ }^{1}$

This is not a phenomenon unique to the UK. A report looking at the question of enhancing access to human rights across the world identified the sense of disillusionment felt by those who have struggled to establish support for human rights - including by incorporation of human rights into domestic law - when poor or otherwise marginalised communities remain sidelined and powerless and in some cases appear to be even worse off than before. As the report identifies, this has led human rights activists and organisations to ask themselves what else needs to be done beyond law and legal reform, to ensure that rights and entitlements are available and accessible to all. ${ }^{2}$

In that spirit, The British Institute of Human Rights (BIHR), in 2002, commissioned a report, Something for Everyone: The Impact of the Human Rights Act and the Need for a Human Rights Commission, ${ }^{3}$ to consider what steps might be necessary if a human rights culture is to take root in England and Wales. ${ }^{4}$ In particular it looked at the impact of the Human Rights Act on the voluntary sector - often important gatekeepers in enabling people to challenge violations of their rights and seek better, human rights compliant, service provision. The evidence gathered provided a valuable insight, not

1 See Luke Clements "Winners and Losers" (2005) 32 Journal of Law and Society 34-50.

2 International Council on Human Rights, Enhancing Access to Rights, 2004, p.1.

3 Published by The British Institute of Human Rights on 10 December 2002, researched and written by Jenny Watson. Available at http://www.bihr. org/programmes4.html.

4 The project did not consider Scotland where there was already a proposal from the Scottish Executive to establish a Human Rights Commission. 
only into the voluntary sector, but also into the attitude of the public sector with respect to human rights.

The report made a number of findings including:-

- awareness of the Human Rights Act has not in general spread outside the legal field. The report remarked "without more attention paid to the promotion of the Human Rights Act and the principles which lie behind it in a way that makes it accessible to lay people the vicious circle of unresponsive public services which lead to legal challenges cannot be broken". 5

- individual members of staff in public services have no understanding of their responsibilities under the Human Rights Act. The report stated: "The lack of any ongoing concerted promotional strategy for the Act means that staff who provide public services - particularly frontline staff - fail to understand what the Act is, the rights that it contains and the responsibilities that they have to uphold it." 6

- there is little or no understanding of the Human Rights Act as a useful framework for public service providers within which problems can be solved and risk assessed, and within which the needs of individuals in the provision of services can be considered. The report stated: "This may be particularly useful for areas where the rights of one individual may need to be balanced against the rights of others, perhaps leading to restrictions on rights which can be justified using the Act's concept of proportionality. Such a framework could enable public service workers to make difficult decisions about allocation of resources, or protection of vulnerable children or adults, with more confidence."

- there is no single authoritative source of advice and information that could help to shape the development of a human rights culture in the absence of a statutory body that could promote and protect human rights. The report went on to say: "Important principles captured in case law are not, at present, applied across a wider area of work. This prevents the development of good practice." 8

These findings led to a single recommendation that the government should establish an independent body capable of effectively promoting and protecting human rights, and should seize the opportunity presented by the single equality body project to do this by creating a Commission for Equality and Human Rights. This, in fact, foreshadowed the emergence of such a proposed body, as detailed in the Equality Bill (and now Equality Act 2006).

These findings prompted BIHR to think about new ways of tackling the problems identified in its report, Something for Everyone.

5 Something ForEveryone, Executive Summary.

6 ibid.

7 ibid.

8 ibid. 


\section{Piloting a new approach to human rights protection}

Its response was to establish, in January 2003, a new stream of work dedicated to human rights promotion and awareness-raising in the voluntary and public sectors, under the banner Community Outreach Programme and since renamed Human Rights Promotion and Training Programme.

The objectives of the programme were:

- in the voluntary sector, to encourage voluntary and community groups to use the Human Rights Act and other international human rights standards as tools to negotiate and attain better standards of public service for their beneficiaries.

- in the public sector, to encourage public authorities to use the Human Rights Act as a framework for best practice and as a tool for better service delivery.

Underpinning the approach was the idea that human rights are an important means by which to improve society, to make it more humane and better able to cope with the differing needs of the people within it. Also to the fore in the development of this work stream was the idea that equality, dignity and respect are core values that underpin the human rights paradigm, and that these values should be the bridge by which people come to understand the relevance and connection of human rights to the lives of ordinary people in the UK.

Underscoring this model also was the belief that human rights litigation alone would not deliver improved service delivery for excluded people. As noted in Something For Everyone, "[Litigation] is not the only - and probably not the fastest - method of achieving change and, used alone, it creates a false perception of the Act." Three years on from that report, one could put it more strongly. Litigation could never bring about the sort of "social change" required if a human rights culture is to blossom. Litigation will always have its place in establishing certain principles. The challenge is how these principles take root across the whole landscape of public service delivery.

\section{Voluntary sector work}

The work comprises running full day human rights awareness-raising sessions with those voluntary and community organisations that work with vulnerable and excluded people. The sessions are free of charge and usually run in-house. One session may have attendees from one organisation or from a variety of different organisations.

The work originally focused on voluntary and community sector organisations working with four groups in particular ${ }^{9}$ although each area was brought "on line" gradually over a period of time, to allow for networks and specialist knowledge to be developed in each area. Over time BIHR has been able to offer training across most of the full range of social exclusion and human rights.

9 Refugees and asylum seekers, disabled people, people with mental health problems and disadvantaged older people. 
The work was set up as an England-wide project and, for managerial and resource reasons, rather unevenly distributed with $80 \%$ of the training taking place in London, the East and the South East and 20\% throughout the rest of England.

There is no one general profile of an organisation that undertakes human rights awareness-raising. They range from small service user or carer led support, advocacy or advice groups, to sector specific umbrella organisations to large public service providing charities.

\section{Public sector work}

The work with the public sector was launched in earnest in early 2004 to run alongside and complement the work in the voluntary sector. There is a particular focus on those public authorities working with vulnerable people such as social services, housing and education departments of local authorities and NHS Trusts. Some work has also been conducted with central government.

The work is undertaken under contract with the public authority. It may consist of a one-off training day for a particular group of staff, but more usually, and desirably, a comprehensive range of training. BIHR encourages the adoption of a training strategy encompassing front-line staff, managers, equality officers and those in non-executive roles, such as councillors in local authorities. Different approaches are usually required for different groups, in particular for those in senior non-executive positions who may have limited time but play a crucial leadership role within an organisation.

BIHR encourages adoption of a rolling programme of training, usually as part of the training provision offered generally across the public authority. Additionally public authorities are encouraged to think about how induction of new staff will be managed so that human rights will be covered, how staff might have access to refresher or more specialist sessions in the future, how agency staff are trained, and how those managing contracted out services are being covered.

Periodically, through any contract period, BIHR will seek evaluation meetings with representatives of the public authority to allow for two-way feedback, which may then inform the future shape or focus of training. It also allows for BIHR to feedback suggestions, often ideas raised by staff within the public authority during training, of how processes or systems within the public authority might be improved to take account of human rights. Suggestions have included the creation of human rights champions in particular departments, the inclusion of a standing human rights agenda item in team and inter agency meetings, the development of a guide to better decision-making using the human rights framework and inclusion of human rights in checklists relating to decisions, for example, concerning child protection.

\section{The methodology}

The style of training is practical, focusing on the day-to-day situations encountered by the participants. Experience has shown that an overly legal and/or formal approach to the content and training method itself can be counterproductive. It tends to feed the idea that human rights is only for 
lawyers and packages the content in such a way that it is out of the grasp (or perceived to be) of many.

The style of training is also interactive and participative, with emphasis on creating a forum for discussion of problems and good and bad practice which can then be used as the basis for illustrating the operation of human rights principles. This is also why case studies form the basis of much of the learning in a training session, as it gives participants a chance to put human rights principles into practice in situations which are familiar to them. Much of the emphasis is on tuning people into when human rights are engaged rather than turning out human rights experts.

The content of the training consists of an explanation of those human rights standards that are particularly relevant for that audience and how they interact with other domestic law and regulations. Participants usually have little knowledge about the former but a well developed knowledge of the latter. Specifically the integral relationship between human rights and the law relating to equality and anti-discrimination is covered in training.

The standards are explained by reference to subject specific examples which will be familiar to the audience. Case law is used where appropriate to illustrate important principles that have been established in a particular area but the training is not simply an account of case law. Emphasis is placed on giving participants the confidence to apply human rights principles, or at least identify a human rights issue themselves without having to know all the relevant case law. Typically participants are invited to consider, in small groups, a number of scenarios and work out which human rights might be relevant and why. A discussion in the plenary group then ensues, where the reasoning and approach of participants is looked at more closely and guidance is given about the correct approach.

Over the course of the training certain fundamental principles, which are key to understanding the operation of human rights are elucidated and illustrated. The objective is that these principles are firmly understood by the end of the training session as they are particularly important for the development of a proactive, best practice led approach:

- human rights are not all of the same nature, some are absolute, some limited and some qualified;

- the principle of proportionality; and

- the nature of positive obligations.

All the training is accompanied by detailed training materials, to support the participants' knowledge base acquired in the training session and for reference following the training.

Evaluation of the training was identified as being key from the start but BIHR's evaluation techniques continue to improve and develop over time.

An evaluation of the training takes place immediately after the training. Participants are asked to complete evaluation forms so that they can rate all aspects of the training. These evaluations are analysed on a six monthly basis to indicate trends, identify strengths and weaknesses and opportunities for future developments. The trainer also completes their own evaluation to capture reflections about training techniques, the content and structure of the 
session, the prevalence of particular human rights issues in the organisations trained, systematic problems encountered as well as specific examples of good and bad practice.

All organisations are followed up within $6-12$ months of the training to assess its impact on the work of the organisation. However, further thought and work is required to measure more accurately the long term impact of training in such a way as to inform the development of new methodologies and tools to bed down a human rights culture.

\section{The resources}

The work under BIHR's Community Outreach Programme was undertaken initially by one full time member of staff recruited specifically for that purpose. That post was funded for three years by the Community Fund (now The Big Lottery). As the work stream developed it quickly reached maximum capacity and further funding was received from Comic Relief allowing another post to be created in 2004. With the public sector work coming on stream in 2004 a third post was created in early 2005. Funds generated by the public sector work were re-invested in the programme to fund the third post and some of the running costs of the programme.

However, the programme has quickly reached full capacity with training sessions typically being fully booked six months or more ahead. The challenge of sustaining existing levels of funding as well as the need to attract new funding to support the strategic development of the work is an on-going challenge.

As the training style has been particularly important in the success for the programme, it is worth noting the need for particular qualities in those carrying out the training. Trainers not only need to have in depth knowledge of the Human Rights Act, other relevant human rights standards, the law relating to equality and the panoply of other relevant domestic law in particular areas, such as the Mental Health Act and the Children's Act, they also need to be effective trainers and communicators. Finding this combination of skills in one person can be challenging. There appears to be a lack of lawyers or people with legal knowledge who are also able, coherently, to take human rights outside its legal box.

\section{Ways to public service improvement using a human rights approach}

\section{Human rights are a practical set of values which can underpin the design and delivery of better public services}

Public authorities need to adopt a human rights approach to their work not only because it is the law, ${ }^{10}$ but also it can be a catalyst in improving services. The application of human rights principles, not least those relating to dignity, autonomy and respect, can help to improve a service user's experience and quality of care and will inevitably lead to improved outcomes

\footnotetext{
10 Human Rights Act 1998, s.6.
} 
The Prime Minister has spoken about redesigning public services around individual need, abandoning the "one size fits all" approach. ${ }^{11}$ The Human Rights Act framework can assist those who deliver public services to consider how best to meet the needs of vulnerable people and put the individual back at the heart of the service.

The Audit Commission, an independent body responsible for ensuring that public money is spent economically, efficiently and effectively, to achieve high-quality local and national services for the public, has underlined the importance of this approach. It has stated "The [Human Rights] Act was a clear statement of rights that need to be taken into account in the delivery of public services". ${ }^{12}$ It has also expressed concern that public bodies have struggled to make the connection between human rights, equalities and service improvement. It found that, in 2003, 58\% of public bodies surveyed had not adopted a strategy for human rights, and in health this figure was as high as $73 \% .^{13}$

If human rights are to inform better service delivery then they must influence the work of public authorities in a number of ways, alongside, and informed by, human rights training:

- The importance of adopting a corporate approach. There is a temptation for public bodies to respond to human rights in a piecemeal way, usually by responding to adverse case law. The human rights framework needs to be integrated into the corporate systems of public authorities. It does not need to be a separate strategy per se and there will be benefits of a level of integration with strategies dealing with equality, race relations and community cohesion.

- The need for human rights principles to be taken into account in the formation and review of policies and procedures. For example, blanket policies, such as a policy stating that the manual lifting of residents in care should never be allowed, should generally be avoided as they fail to put the individual at the centre of decision making.

- The need for human rights principles to inform decision making including decisions made by front-line staff who deliver services in practice, for example care staff in a residential care home.

Some organisations have very helpfully provided guidance to assist people in adopting a human rights approach when making policies or taking decisions including a number of checklist models. ${ }^{14}$ The development of desk based learning tools and other guides to help with decision making is also underway. ${ }^{15}$

11 Speech by the Prime Minister about Public Service Reform, 25 January 2002. http://www.number-10.gov.uk/output/Page3008.asp.

12 Human Rights: Improving Public Service Delivery, Audit Commission, October 2003, p.3.

13 ibid., p.7.

14 The most comprehensive is to be found in Human Rights Act Toolkit, Jenny Watson and Mitchell Woolf, Legal Action Group, 2003.

15 See also, e.g. the guide published in July 2005 for non executive board members of NHS Trusts on promoting equality and human rights in the NHS. Available at 


\section{Northern Ireland Legal Quarterly [Vol. 57, No. 1]}

There are examples of changes of practice, but more research is needed to gather quantitative and qualitative information about the impact of incorporating a human rights approach into the way public services are delivered. An evidence base needs to be amassed in order to evaluate "what works".

The Audit Commission provides twelve examples of changes made by public bodies to their policies and practices including:

- A borough council improving its procedures for appeals by appointing an independent chair.

- A number of councils' standard contracts and grant conditions have been amended to ensure that human rights issues are clearly delineated.

- A health trust conducting policy review of consent and resuscitation policies, violence and aggression policies, selection and recruitment and disciplinary policies, patient control and restraint policies. ${ }^{16}$

BIHR also comes across changes in policies and procedures some of which are similar to those uncovered by the Audit Commission, or was able to suggest changes in evaluation meetings with public authorities, some of which were mentioned above. BIHR was also made aware, through its evaluation process, of changes in practice at the front line of service delivery:

- A disabled man questioning the practice by which he was examined naked in front of different people a number of times, a situation which he found humiliating.

- Challenging the conditions of seclusion for mental health patients in a secure unit.

- The review of a decision in a residential care home that bed pans would not be provided between lunch and tea time.

A recent paper on improving public services using a human rights approach noted "Implementation beyond paper compliance to actual delivery is always the mountain that needs to be shifted and it is likely to require systematic planning and fundamental systemic reform led from the top. . ."17 The improvement of public services by way of the adoption of a human rights framework for delivery will depend, not only on training, but also partly on the overall culture of the public body concerned, including how the staff themselves are treated. In well run and "healthy" public authorities the incorporation of a human rights approach should complement what the organisation is already doing. ${ }^{18}$

http://www.dh.gov.uk/PolicyAndGuidance/EqualityAndHumanRights/fs/en (accessed November 05).

16 Human Rights: Improving Public Service Delivery, Audit Commission, October 2003, p.14.

17 Frances Butler, Improving Public Services: Using a Human Rights Approach, Institute for Public Policy Research, June 2005, p.42.

18 ibid. 


\section{Open textured nature of human rights makes it well suited to underpinning difficult decisions faced by public authorities where a number of interests need to be taken into account}

Many of the rights in the Human Rights Act are qualified rights and are concerned with ensuring that the rights of the individual are balanced against the rights of others and the interests of the community. Even those rights which are absolute in nature, like the right not to be subjected to torture, inhuman or degrading treatment, involve consideration of the particular circumstances in order to ascertain whether the right has been engaged at all.

Application of the human rights framework therefore usually means the application of a decision making process, within certain parameters, which is objective and has considered and balanced, using the concept of proportionality, the rights of all involved. This is inherently uncomfortable for some who desire certainty - these are people who in training will want to know "the answer" rather than understand and apply "the process". It is however precisely because this is the nature of the human rights paradigm that it is so suitable for providing a framework for best practice development across a broad spectrum of public services.

\section{A human rights approach to decision making can help to achieve transparent, objective and well reasoned decisions}

Because, as outlined above, application of the human rights framework is as much about how one applies the standards as the standards themselves, it allows for decisions to be made that are objective and transparent. This helps to show service users and others that the decision making process is objective and helps protect an organisation from allegations that it has not considered people's rights.

\section{The role of the voluntary sector}

The voluntary sector has an important role to play in driving up standards of public service delivery to vulnerable people. It has a crucial influencing and representative role in advancing the interests of others. Voluntary organisations can be effective in holding public authorities to account for their legal responsibilities and bad practice by using preventative mechanisms and advocacy which may or may not include legal action. This has been powerfully demonstrated by those voluntary sector organisations which BIHR has trained over the past three years. ${ }^{19}$

\section{The role of inspectorates}

There is a "sharp edge" to human rights promotion. Promotion of human rights standards has a direct link with protection of human rights when aimed at those who have the capacity to affect the enjoyment of human rights. That is why the inspectorates with responsibility for inspecting public services are increasingly aware that a service which is delivered within a human rights framework is more likely to be a quality service which is responsive to service user need.

19 Frances Butler, Human Rights: Who Needs Them? Using Human Rights in the Voluntary Sector, Institute for Public Policy Research, 2004. 


\section{Northern Ireland Legal Quarterly [Vol. 57, No. 1]}

In October 2005 the Audit Commission launched a new form of Comprehensive Performance Assessment for single tier and county councils. ${ }^{20}$ One of the key lines of enquiry by which a local authority will be inspected is its capacity to deliver what it is trying to achieve. The "criteria for judgement" include whether the council has a strategic and integrated approach to diversity, human rights and user focus evident in its policy development, employment practices and service delivery and whether the council promotes these to its staff, partners and the wider community. Other inspectorates are gradually taking on board the importance of human rights in improved service delivery outcomes and therefore considering how this might inform the inspection process. ${ }^{21}$

\section{Challenges for the future}

The work of BIHR will be useful to the new Commission for Equality and Human Rights in having tested out some approaches to human rights promotion and the promotion of best practice in this area, two of the proposed human rights duties of the new body.

Through its work it is possible to identify where some of the significant challenges will lie in developing a strategy aimed at promoting human rights awareness and good practice. Notably, while recognising the importance of training, there is a huge challenge in making this "work to scale" given the large number of public authorities which need to engage with this agenda. When thinking about this, consideration will need to be given to the importance of cross-sector working, the need for engagement of frontline staff, managers and those with senior executive and non-executive roles and the need for messages to be practical, relevant and integrated with those on equality. Capacity within public authorities will need to be built and supported in order for the public bodies themselves to become the drivers in developing a human rights culture. Co-ordinated work with both the inspectorates of services and the voluntary sector will be an important component of any strategy given their ability to challenge bad practice and hold the providers of public service to account. Given the wide range of services that are contracted out by public authorities, the private and voluntary sector will also need to be engaged as providers of services themselves.

${ }^{20}$ CPA - The Harder Test. This can be found at http://www.audit-commission. gov.uk/cpa/index.asp?page=index.asp\&area=hpcpa.

21 Frances Butler, Improving Public Services: Using a Human Rights Approach, Institute for Public Policy Research, June 2005, p.26. 